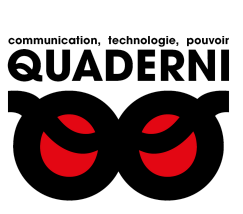

\title{
Quaderni
}

Communication, technologies, pouvoir

\section{Le « complotisme 2.0 », une étude de cas de vidéo recombinante : Alain Soral sauve Glenn et Tara dans The Walking Dead}

\section{Julien Giry}

\section{(2) OpenEdition \\ Journals}

Édition électronique

URL : https://journals.openedition.org/quaderni/1112

DOI : 10.4000/quaderni. 1112

ISSN : 2105-2956

Éditeur

Les éditions de la Maison des sciences de l'Homme

Édition imprimée

Date de publication : 5 octobre 2017

Pagination : 41-52

Référence électronique

Julien Giry, «Le " complotisme 2.0 », une étude de cas de vidéo recombinante : Alain Soral sauve Glenn et Tara dans The Walking Dead », Quaderni [En ligne], 94 | Automne 2017, mis en ligne le 05 octobre 2019, consulté le 22 juillet 2021. URL : http://journals.openedition.org/quaderni/1112 ; DOI : https:// doi.org/10.4000/quaderni. 1112 


\section{$D$ ossier}

Le "complotisme 2.0", une étude de cas de vidéo recombinante : Alain Soral sauve Glenn et Tara dans The Walking Dead

Si le cinéma, la télévision, les jeux vidéo dans les années 1980, $1990^{1}$ et l'internet depuis les années 2000 constituent de formidables machines à diffuser, à désenclaver et à légitimer les croyances conspirationnistes, il serait faux de dire que ces nouvelles technologies de l'information et de la communication ont créé le phénomène conspirationniste du $\mathrm{XXI}^{\mathrm{e}}$ siècle. En effet, si l'internet en particulier a joué un incontestable rôle, à la manière d'une " cyber-caisse » de résonance, d'interface spatio-temporelle accélérant la diffusion des thèses conspirationnistes, la mise en relation des adeptes du complot et la création de réseaux et groupes complotistes planétaires, il n'en demeure pas moins que le phénomène social et politique conspirationniste préexistait aux NTIC qui ne sont en fait qu'un moyen de diffusion des théories du complot dans l'opinion publique.

\section{Julien \\ Giry}

Docteur en science politique

CNRS IREMAM

Chercheur associé à IDPSP Université de Rennes 1
Toutefois, il semble que l'internet soit bien plus qu'un simple moyen parmi d'autres dans la mesure où il est devenu le moyen prioritaire et pratique de produire et diffuser immédiatement, à la fois quasi instantanément et (presque) sans médiation, à l'échelle planétaire des messages conspirationnistes. Ainsi, en 2016, Dieudonné déclarait :

«L'internet est un espace messianique fou pour moi, c'est grâce à l'internet que j'ai pu continuer mes activités sur scène et plus largement m'émanciper de l'ancien monde [...] on a un outil qui est à la fois un espace qui s'est ouvert, infini, qui est l'internet avec le meilleur et le pire de l'histoire de l'homme. Je pense qu'ils l'ont laissé se mettre en place parce qu'ils nous contrôlent un peu mieux chaque jour, mais nous les contrôlons 
aussi un peu mieux et nous contrôlons surtout qui ils sont, leur action [...] Je crois qu'on aura accès à tout puisque maintenant on a l'outil qui le permet. Le monde entier va changer [...] J'ai survécu grâce à cet espace incroyable de l'internet, c'est formidable [...] c'est plus des stratégies de propagande qui fédèrent, les gens vont là où ils ont envie d'aller ${ }^{2}$ "

De ce point de vue, l'internet a pu être largement perçu, et bien au-delà des cercles conspirationnistes d'ailleurs, comme un espace social de liberté, de démocratie directe ou « radicale $^{3}$ » accomplie, voire même encore de « contre-démocratie $^{4}$ » à côté du monde réel, doté de ses propres codes, symboles, représentations et imaginaire collectif; et c'est l'une des raisons pour lesquelles l'internet est devenu le lieu privilégié de l'expression des théories du complot. En effet, par son dynamisme et sa souplesse, l'internet permet, à peu de frais, et sans qu'il faille disposer de hautes compétences techniques, à chaque internaute de diffuser, de créer ou de remodeler à l'infini des contenus afin de générer un espace d'expression alternatif. Chacun, via Twitter par exemple, peut se faire média en diffusant, répercutant ou créant des informations, des e-rumeurs, des billets d'humeur ou tout autre énoncé performatif. De ce fait, l'internet participatif, ou 2.0, en tant que dispositif sociotechnique est devenu une forme de sociation politique qui offre aux acteurs des lieux de rencontres et d'expressions « ethnosociologiques $^{5} »$, complotistes parfois, pour des savoirs alternatifs et/ou en rupture avec ceux des dominants ${ }^{6}$. Alors, si un tel espace de liberté, du reste largement fantasmé, ne peut qu'être loué, à condition toutefois de ne pas tomber dans l'angélisme naïf d'un néo-scientisme post-matériel ${ }^{7}$, il n'en demeure pas moins que celui-ci va être, en partie au moins, capté et détourné par de nombreux acteurs, $y$ compris conspirationnistes.

«Internet a ouvert un espace pour des formes d'expertise publique portées par des individus ou des collectifs de tous ordres. Le web participatif contribue au développement d'une contre-expertise et à celui de pratiques d'investigation qui associent journalistes professionnels, amateurs et internautes ${ }^{8}$ »

Avec l'internet, nous assistions en effet à un nivellement épistémologique, où le "savoir des gens ${ }^{9}$ » concurrence les savoirs des savants, sachants et/ou experts qui se traduit par une « resourcisation » de l'incompétence des gens ordinaires, capables de découvrir la "vérité vraie » à la manière de " détectives ${ }^{10}$ » ou de

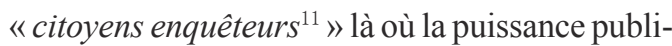
que, incarnée par la figure type du policier ${ }^{12}$, et ses agents, complices ou simples idiots utiles du « système », en sont incapables et non disposés. Or, cette recherche de la " vérité vraie » passe depuis les années 2000 par la diffusion de vidéos en ligne. Par exemple, on pense aux vidéos où Richard Gage ${ }^{13}$, l'un des fondateurs du Mouvement pour la Vérité autour du 11 septembre, explique à l'aide de boîtes en carton à l'écroulement aléatoire l'impossibilité de la "version officielle » des attentats. Selon lui, il s'agirait en réalité d'une démolition contrôlée. Soulignons toutefois que cette « démonstration » se fait au plus grand mépris de toutes les lois de modélisation et d'architecture en matière de maquette ${ }^{14}$.

Quoi qu'il en soit, jusqu'alors parent pauvre de l'internet pour des raisons techniques, nous 
assistons depuis 2001 à « un mouvement massif de diffusion de vidéos militantes sur l'internet que l'on pourrait qualifier de "tournant vidéo de l'internet militant"15 $»$. Or, ce tournant du médiactivisme se caractérise par le développement de montages intertextuels empruntant aussi bien aux images diffusées dans des reportages ou des documentaires télévisés, des images d'archives, des dessins animés que des extraits de films, de chansons ou de discours. Ce phénomène, connu sous le nom de found footage, rendu facile par les outils informatiques très accessibles ${ }^{16}$, engendre alors, par le (re/dé)montage de séquences éparses, la création d'un nouvel imaginaire, d'une nouvelle unité de sens mélangeant fiction et réalité, romantisme et horreur ; le tout présentant bien souvent un certain esthétisme graphique et sonore mais aussi un contenu ambigu. Ainsi, pour le chercheur québécois André Habib, le found footage consiste dans :

"Le réemploi d'images (films publicitaires, images de séries $B$, films des premiers temps, images d'archives) qui tend à les remettre en lumière, nous invitant à les "revoir » et à les faire signifier autrement, selon les nouvelles chaînes dans lesquelles elles s'inscrivent et le traitement particulier (ralenti, recadrage) auquel elles donnent lieu. [...] Ces reprises déploient une intelligence critique, se fondent sur un plaisir esthétique ou simplement ludique. En détournant le sens initial des images et en exposant ainsi leur fonction première, ces reprises nous invitent également à nous arrêter sur ces images, à les regarder pour " ce qu'elles sont » : des artefacts culturels, des traces de l'histoire, des fragments de pellicule. En d'autres mots, la pratique du found footage peut être analysée comme une modalité de l'arrêt sur image (même si, dans les faits, l'image est animée) entendue comme un mode de repotentialisation et de dévoilement des images, parfois oubliées, souvent invisibles ${ }^{17}$ »

Ainsi entendues, les vidéos recombinantes peuvent être considérées comme « une méthode d'exposition de formes d'expressions hétérogènes et comme un dispositif qui préside aux lois de déconstruction et de reconstruction de l'œuvre d'art ${ }^{18}$ »

Dès lors, nous proposons de réaliser une étude de cas autour d'une vidéo recombinante française qui nous a semblé exemplaire du phénomène : Alain Soral sauve Glenn et Tara dans The Walking Dead, abrégée par la suite en Soral sauve. Dans un premier temps, nous procéderons à une description factuelle de la vidéo ainsi que de ses conditions d'énonciation et de production. Nous procéderons ensuite à l'analyse politique/politologique de cette vidéo en montrant comment elle s'inscrit symboliquement dans l'imaginaire conspirationniste de Soral au point d'en constituer une forme condensée. Enfin, il nous semble indispensable de présenter dans une ultime partie les usages contenus dans la vidéo tant ils sont typiques du found footage.

\section{Soral sauve : la vidéo en elle-même et son cadre d'énonciation}

Postée sur YouTube le 28 mars 2016 par Buzz Man, Soral sauve ${ }^{19}$ s'inscrit dans une série de quatre montages, tous postés par Buzz Man, mettant en scène Alain Soral gagnant un oscar ou terrorisant l'humoriste Norman par exemple. D'une durée d'une minute quarante secondes, 
la vidéo totalise en avril 2017 un peu moins de 35000 vues et a fait l'objet, dès le jour de sa diffusion, d'un fil de discussion sur la plateforme jeuxvideo.com lancé par Falcom ${ }^{20}$, alias Buzz Man sur YouTube, l'auteur de la vidéo. Les avis des internautes sont dithyrambiques tout au long des neuf pages de commentaires. L'approbation générale s'exprime soit sous la forme de l'émoticône « mort de rire », soit au travers de formules telles que «bordel, c'est trop bon», «putain, sympa !!!», ou encore «énorme! ». Un commentaire, plus politisé, propose même une référence explicite au slogan, « Gauche du travail, Droite des valeurs », du mouvement présidé par Soral, Égalité \& Réconciliation (ER), lorsque Wannachill5 écrit : « les droites des valeurs que se mangent les zombies! ». De même, pour Alexis Secret sur YouTube: "L'enchaînement droite des valeurs, gauche du travail était trop puissant pour eux ». C'est d'ailleurs en raison de son caractère politique faisant sens vers l'univers conspirationniste de Soral au travers de « signes disséminés [qui] renvoient à une vérité sousjacente $^{21} »$ que nous avons retenu cette vidéo. Du reste, il semble que la dimension politique de la vidéo n'a pas échappé à Soral lui-même dans la mesure où, dès le lendemain (le 29 mars 2016), elle fut reprise, ainsi que les trois autres, par son site internet dans une page intitulée Attention les imposteurs SuperSoral arrive !. Les vidéos y sont ainsi présentées :

"Il y a les vrais, et les faux héros. Le faux héros est celui que le Système tente de nous vendre, avec de plus en plus de mal, chaque jour que Dieu fait. Et puis il y a le héros populaire, dont le peuple se sent spontanément proche, mais qui n'a en général ni les faveurs ni les honneurs de l'élite. [...] Dans ces quatre petits montages humoristiques trouvés sur le net et réalisés par des inconnus, Alain Soral apparaît tantôt comme le sauveur de l'honneur bafoué des gens, le représentant des humiliés et des oubliés, tantôt comme la terreur des fausses élites, celui qui brise le spectacle de l'entre-soi. [...] Conclusion? La fausse élite - ou élite imposée - ne peut pas tenir indéfiniment, même si elle dispose de toutes les armes possibles (argent, politiques, médias, police, justice ${ }^{22} »$

Quant au montage intertextuel lui-même, Soral sauve est composée de trois formes et types de contenus différents immédiatement identifiables pour les jeunes générations. Par ordre d'apparition, nous retrouvons d'abord, jusqu'à la $56^{\mathrm{e}}$ seconde, des images et la bande son (version française) tirées de la série télévisée The Walking Dead ${ }^{23}$. La première séquence proposée nous montre Glenn (Steven Yeun), un jeune asiatique, et Tara (Alanna Masterson), deux personnages phares de la série, acculés dans une sorte de grotte sombre par une horde de zombies inamicaux. Apeurés et à court de munitions, les deux adolescents se préparent à une mort aussi certaine qu'horrible lorsque, soudain ( $53^{\mathrm{e}}$ seconde), une musique inattendue retentit : il s'agit du thème d'entrée sur le ring du catcheur John Cena ${ }^{24}$. S'ouvre alors la deuxième séquence (de la $57^{\mathrm{e}}$ seconde à 1,06 min) qui, sur ce fond musical, voit apparaître en très gros plan et en contre jour Alain Soral, yeux bleus scintillants. La troisième séquence $(1,07 \mathrm{mn}$ jusqu'à 1,28$)$ entremêle ensuite des images de Soral effectuant des mouvements de boxe et des images de la série où les zombies reçoivent, jusqu'à leur extermination totale, 
des balles dont le tireur est hors du champ de la caméra. À travers le montage, tout se passe comme si les balles sortaient symboliquement des poings de Soral. L'avant dernière séquence $(1,29$ à 1,37$)$ propose des images où Glenn et Tara apparaissent sains et saufs mais, comme sonnés, hagards et aveuglés par la lumière du jour qui tranche avec les ténèbres de la première séquence. Enfin, les trois dernières secondes de la vidéo insistent sur un plan moyen de Soral satisfait et en pleine lumière.

Ainsi se décompose donc cette vidéo à forte charge symbolique qui mélange des images issues de la série télévisée The Walking Dead, d'autres tirées d'un film de propagande soralien et la musique de Cena, dont il convient à présent de procéder à l'analyse politique et politologique.

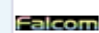

\section{Falcom \\ 29 mars 2016 à 00:18:45}

\section{"Raph Raphil y a 6 minutes}

A mon humble avis, les marcheurs sont une exacerbation métaphorique du consommateur athée occidental soumis à ses maîtres atlantistes et paratisés par les dogmes judéo-maçonniques depuis les bancs de l'école, et ça c'est pas moi qui le dit hein. D'ailleurs on voit que ces "zombies" (néologisme bâtard créé de toutes pièces par les élites mondialistes) anthropologiquement c'est des merdes, des mutants. Si on pousse un peu plus la comparaison, on observe dans la série que les premières victimes sont les habitants des grandes villes. Nombreux ont été les gens à avoir été piégé dans les embouteillages, et ça Soral nous l'avait prédit hein il en parle dans ses livres.

En face d'eux se dresse Soral qui les repousse à l'aide seule de sa force de travail, ses poings, contrairement à cette petite fiotte asiatique au nom américanisé incapable de se défendre sans pistolet, merci l'abrutissement des masses par les lobbies hein. Ah c'est son grand-père vietkong qui a repoussé l'impérialiste américain jusqu'au coeur de sa jungle natale qui doit se retourner dans sa tombe."

Très bon

\section{Soral sauve : une concentration de l'imaginaire conspirationniste soralien}

Comme l'atteste cette capture d'écran issue du fil de discussion Soral sauve de jeuxvideo.com, il est manifeste que le caractère symbolique, allégorique et métaphorique de la vidéo est largement compris par Raph Raphil, dont Falcom reprend le post, lorsque, à travers un pastiche de la phraséologie et de l'élocution soraliennes, il la réinscrit dans l'imaginaire complotiste de Soral. Et, comme le montrent les captures d'écran des commentaires postés et « likés » sur YouTube, d'autres contributeurs ne s'y trompent pas non plus :

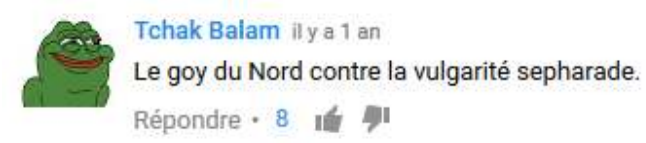

johanna baranov il y a 9 mois

les zombies talmudo sioniste non pas résisté a une mitraillette $40 \%$ eleno chretienne. Répondre 1 II ly 


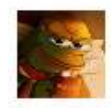

Potato Sack il y a 1 an

La pudeur hélléno-chrétienne est trop puissante

Répondre 36 it 91

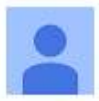

il $y$ a 1 an

Mitraillette goy $40 \%$

Répondre 32 if 91

DZ AMAZIGH ily a 1 an

la puissance de ce goyim est redoutable

"haziza fait attention"

Répondre $\cdot 27$ if 91

Vansco 1000 ily a 1 an

La puissance de ce goy du nord

Répondre - 25 if 7

De plus, comme nous l'avons déjà souligné, le fait que ER relaye cette vidéo traduit la validation de son contenu par Soral lui-même.

Dans l'imaginaire conspirationniste de Soral, Glenn et Tara incarnent dans le montage la multitude ignorante, la foule apeurée et aveuglée, dominée et incapable de se défendre par elle-même. Leur caractère américain et leur absence de virilité, une femme et une «petite fiotte asiatique ", en font en outre des figures types des détestations soraliennes qui stigmatisent sans relâche « le lobby gay " ou "le féminisme hystérique » qui s'opposent dans cette logorrhée manichéenne sans sophistication aux valeurs « helléno-chrétiennes » masculinistes ${ }^{25}$. Toujours au rayon des métaphores symboliques, les zombies incarnent la figure centrale de l'ennemi au sein de l'imaginaire politique de Soral : la domination inique du «lobby sioniste », parfois rebaptisé « communauté organisée dont il faut taire le nom », "communauté de lumière » ou encore «communauté qui a souffert plus que les autres ». Surtout, le processus de déshumanisation de l'ennemi, réduit ici à une multitude anonyme, agressive et sanguinolente faite de lambeaux de chair, dépourvue de conscience ou de sentiments moraux, est typique de la représentation des boucs-émissaires dans les mythes conspirationnistes $^{26}$.

À l'opposé, face à la multitude ignorante et la minorité usurpatrice, Soral est mis en scène, y compris par les effets de caméra (gros plan sur les yeux bleus, lumière étincelante/ténèbres), comme un héros authentique et solitaire dans son combat inlassable contre les forces du mal numériquement supérieures. On retrouve ici une autre thématique récurrente de la littérature complotiste ou antisémite où un homme juste, mais seul, révèle le complot au grand jour ${ }^{27}$. Or, c'est avec ses seuls poings, ses «droites des valeurs et ses gauches du travail », que le héros solitaire terrasse ses innombrables ennemis. Ici encore la charge symbolique est puissante : les mains, les mains nues, les mains sans artefact, les « mains propres et [la] tête haute ${ }^{28} »$ des leaders populistes, symbolisent à la fois la pureté du combat authentique débarrassé de tous les artifices et son ancrage en faveur de la cause de « vrai » peuple laborieux, celui des dominés droits, courageux et honnêtes face à la «fausse élite - ou élite imposée - [qui] ne peut pas tenir indéfiniment, même si elle dispose de toutes les armes ${ }^{29}$ possibles (argent, politiques, médias, police, justice) ${ }^{30} »$. Enfin, la mythification symbolique de Soral au rang de héros/héraut (celui qui porte les messages importants) passe par le thème musical mobilisé qui le classe irrémédiablement du côté du Bien. 
En effet, dans le panthéon des personnages archétypaux du catch, Cena est le face (gentil) par excellence. Surnommé The Marine ou The Champ', Cena personnifie le héros patriote indestructible et droit, toujours prêt à voler au secours de ses camarades les plus faibles ou dans la difficulté. Ne renonçant jamais à s'engager physiquement et moralement dans un combat qui semble pourtant perdu d'avance tant le déséquilibre des forces est manifeste, le face est celui qui par sa seule détermination et son seul courage renverse les montagnes. En retour, il est adulé par le public, le peuple, qu'il défend et représente symboliquement $^{31}$. Et, dans la chanson comme dans la vidéo, une série d'oppositions entre « eux » (le mal) et « nous/je » (le bien) est savamment orchestrée. Par exemple : «Your time is up, my time is now » (votre temps est terminé, mon temps commence) ou «I got my soul straight » (mon âme est droite) / « they hate » (ils haïssent).

En somme, cette vidéo recombinante synthétise les traits essentiels de l'imaginaire complotiste de Soral au travers de métaphores et de représentations symboliques politiquement signifiantes : le héros pur et courageux luttant seul (Soral, la musique de Cena) face à un ennemi omnipotent, immonde (littéralement im mundus - hors du monde) et déshumanisé (les zombies / le lobby sioniste) afin de délivrer du joug oppresseur le peuple aux abois (Glenn et Tara).

\section{Soral sauve : une vidéo recombinante type}

Outre son contenu politique manifeste, cette vidéo a également retenu notre attention car elle nous a semblé paradigmatique de la technique et des usages du found footage qui permet de décon- textualiser, et en même temps de recontextualiser, des images et des sons, et pourquoi pas des textes ou toutes autres formes de contenus, afin de leur donner une nouvelle unité de sens, ouvertement militante et subjective, en opérant des variations et un épuisement des contenus originels ${ }^{32}$.

Ainsi, dans Soral sauve, un support visuel, la série The Walking Dead, et audio, The Time is Now, sont totalement extraits de leurs contextes originaux de production et de diffusion, le divertissement et la fiction télévisée et sportive, afin de leur octroyer une signification politique faisant sens de manière esthétisée dans l'imaginaire complotiste de Soral. Cette esthétisation passe d'abord par un « usage matériologique ${ }^{33}$ » typique du found footage qui se traduit dans la vidéo étudiée par la diversité et la succession de contrastes chromatiques et de plans caméra opérés par le montage. Or, comme le remarquent Machin et Jaworski, si les supports mobilisés rendent généralement l'espace social mis en scène « visuellement plus attrayant et distrayant, [ceux-ci] proviennent d'un monde pré-structuré idéologiquement $^{34} »$. En d'autres termes, avec le développement des vidéos recombinantes, parce qu'elles s'inscrivent dans un cadre idéologique pré-établi, on peut plaider en faveur d'un usage tactique, au sens clausewitzien, de l'internet dans la mesure où les auteurs se réapproprient de manière subversive ou pour mieux les dévoyer les codes et les représentations de la communication visuelle $\mathrm{e}^{35}$. Ainsi, pour Brenez, cet « usage critique $^{36} \gg$ se matérialise par le détournement visuel et sonore opéré par les auteurs de vidéos recombinantes dans leur manière de s'approprier et de rendre subjectifs les contenus ré-assemblés pour les faire coïncider avec des représentations 
sociopolitiques pré-structurées ou pré-établies ; dans le cas d'espèce celles de l'imaginaire conspirationniste de Soral. Autrement dit, les auteurs/ diffuseurs de vidéos recombinantes font non seulement varier le sens des matériaux sonores et visuels originaux mobilisés, mais encore ils en épuisent leurs caractères intrinsèquement polysémiques pour leur conférer une signification unique et immédiatement saisissable par le(s) public(s), ciblé(s) ou non, comme en attestent les commentaires cités précédemment.

Or « dans un environnement où le virtuel domine de plus en plus », en particulier dans le cadre du fait social et politique complotiste, " je trouve ce procédé dangereux [parce qu'il] induit une sorte de négation du référent qui peut semer le doute dans les esprits et conduire à toutes les falsifications : après tout, cette actualisation des images n'est rien d'autre qu'une forme de manipulation ${ }^{37}$ ». Aussi, en "réagencement et resignifiant des séquences puisées dans des sources diverses ${ }^{38} "$, les vidéos recombinantes participent d'un brouillage entre les faits et la fiction, "l'énoncé référentiel [et] l'énoncé fictionnel ${ }^{39}$ ", alors même que ces « deux genres sont irréductiblement séparés et s'opposent même radicalement $t^{40} \gg$. Surtout, faute d'une éducation à la critique des médias et des images suffisamment développée, «nous ne pouvons pas présumer que les spectateurs liront ces éléments comme une grammaire symboliquement connotée ${ }^{41} "$, subjective et idéologiquement (pré)constituée et non comme la «vérité vraie » se donnant à voir en elle-même au travers des images ${ }^{42}$.

Dans un monde saturé d'images qui se succèdent à un rythme effréné, l'internet, qui se veut un diffuseur infini, offre potentiellement à la planète entière un accès à une base de données quasi illimitée ${ }^{43}$. Ainsi, la création d'archives du web, le développement des podcasts, l'avènement de plates-formes de téléchargement en peer to peer ou de sites de partage de contenus nous permettent de piocher à souhait dans des millions de contenus audio, vidéos ou textuels disponibles afin de les recombiner de manière infinie. Or, face à un tel fourmillement, et notamment lorsque des acteurs radicaux s'emparent du web et de ses potentialités afin de diffuser leurs discours dans des formes esthétisées et idéalisées, il est plus que jamais impératif de développer une véritable éducation à la critique des médias conçue comme autant d'outils à disposition du public pour comprendre les enjeux et usages sociopolitiques de la communication (visuelle) moderne. Ceci est tout particulièrement indispensable lorsqu'elle passe par des dispositifs sociotechniques tellement présents dans nos vies quotidiennes que, parfois, nous ne prenons même plus la peine de les interroger. 
$\mathrm{N} \cdot \mathrm{O} \cdot \mathrm{T} \cdot \mathrm{E} \cdot \mathrm{S}$

1. Peter Knight, Conspiracy Culture. From Kennedy to the X-Files, New York, Routledge, 2000. Samuel Chase Coale, Paradigms of Paranoia: The Culture of Conspiracy in Contemporary American Fiction, Tuscaloosa, University Alabama Press, 2005. Gordon B. Arnold, Conspiracy Theory in Film, Television and Politics, Westport, Praeger Publishers, 2008.

2. Dieudonné M'Bala M'Bala, « L'heure la plus sombre »,ER FM, n²8, 14 mars 2016. Accédé : 15 avril 2017. En ligne :http://www.egaliteetreconciliation.fr/LHeure-la-plus-sombre-du-14-mars-2016-Dieudonneet-Alain-Soral-38331.html

3. Benjamin Loveluck, «Internet, vers la démocratie radicale ? », Le débat, ${ }^{\circ} 151,2008$, pp. 150-166.

4. Pierre Rosanvallon, La Contre-Démocratie. La politique à l'âge de la défiance, Paris, Seuil, 2006.

5. Anita M. Waters, « Conspiracy Theories as Ethnosociologies. Explanation and Intention in African Americain Political Culture », Journal of Black Studies, vol.28, n¹, 1997, pp. 112-125.

6. Cette concurrence entre " profanes » et « experts » est illustrée par l'avènement du journalisme participatif ou citoyens, notamment grâce à des dispositifs sociotechniques tels que Twitter. Ainsi en témoigne Eric Fottorino, ancien directeur du Monde: «Avant, l'AFP, c'était la source, la lumière zénithale de l'information. L'info tombait de l'AFP. Maintenant, c'est l'AFP qui court derrière le tweet [...] L'information est devenue une conversation dans laquelle les professionnels de l'information sont une partie de la conversation mais pas toute la conversation. Et on a donc cette horizontalité, alors qu'avant, il y avait ceux qui savaient et ceux qui ne savaient pas. Il y avait cette verticalité [...] À partir de là, c'est vrai que la perte de monopole de l'information par les journalistes les oblige à rentrer dans cette conversation. Et quand il s'expose, quand il entre dans le monde de Twitter ou de Facebook, ça veut dire qu'il accepte le reproche, d'être corrigé, démenti, d'être complété. Il doit accepter qu'il y ait une réactivité qui va le remettre en cause d'une certaine manière. Alors que, jusque-là, la ligne verticale faisait que le lecteur achetait son journal, le lisait et gobait ça ». Cité in Antoine Dubuquoy et Nico Prat, Twittus Politicus, Montrouge, Fetjaine, pp.92-93. 7. À l'inverse, une étude américaine menée par Lawrence, Sides et Farrell à propos des lecteurs de blogs politiques a montré que près de $94 \%$ des 2312 personnes interrogées consultent uniquement des blogs épousant leurs vues. Loin d'un espace ouvert où chacun aurait accès à une multitudes d'informations pluralistes, l'étude tend à démontrer que sur le net, les acteurs sociaux se replient à la manière d'un entresoi cognitif sur des espaces confirmant et renforçant leurs opinions en leur donnant même l'illusion d'être majoritaires. Les auteurs parlent alors d'homophilie. Eric Lawrence, John Sides et Henry Farrell, « SelfSegregation or Deliberation ? Blog Readership, Participation and Polarization in American Politics ", Perspectives on Politics, vol.8, n², 2010, p. 146.

8. Dominique Cardon, Fabien Granjon, Médiactivistes, Paris, Presses de Sciences Po, 2013, p.122

9. Michel Foucault définit le « savoir des gens » comme «la réapparition du savoir d'en dessous, de ces savoirs non-qualifiés, de ces savoirs mêmes disqualifiés : celui du psychiatrisé, celui du malade, celui de l'infirmier, celui du médecin, mais parallèle et marginal par rapport au savoir médical [...] et qui n'est pas du tout un savoir commun, mais, au contraire, un savoir particulier, un savoir local, un savoir différentiel, incapable d'unanimité ». Michel Foucault, Dits et Écrits II, 1976-1988, Paris, Gallimard, 2001, p. 164. 10. Luc Boltanski, Énigmes et complots. Une enquête à 
propos d'enquêtes, Paris, Gallimard, 2012, pp. 56-60. 11. Kathryn S. Olmsted, « The Truth is Out There : Citizen Sleuths From The Kennedy Assassination to The 9/11 Truth Movement », Diplomatic History, vol.35, n 4 , 2011, pp. 671-693.

12. Dominique Kalifa, « Policier, détective, reporter. Trois figures de l'enquêteur dans la France de 1900 », Mil neuf cent. Revue d'histoire intellectuelle, vol.1, n²2, 2004, pp. 15-28.

13. Par exemple: « 11 septembre 2001 - Richard Gage, « comment expliquer cela? »», YouTube, 2 avril 2016. Accédé : 14 avril 2017. En ligne : https://www.youtube. com/watch? $\mathrm{v}=$ RLzoMRvO0aw

14. Jean-Pierre Muzeau, « Vous avez dit démolition contrôlée ? », Science et pseudo-sciences, hors série, n²96, juin 2011, pp. 38-41.

15. Olivier Blondeau, Devenir média. L'activisme sur internet, entre défection et expérimentation, Paris, Éditions Amsterdam, 2007, p. 61.

16. Ainsi, Apple propose à ses clients le logiciel Imovie qui permet de réaliser des montages tandis que Microsoft développe Windows Movie Maker. D'autres logiciels sont également disponibles parmi lesquels Adobe Premier Pro, Sony Vegas Pro pour les logiciels sous licence ou OpenShot Video Editor, VideoMan Movie Creator pour les logiciels libres.

17. André Habib, «Les stases de l'histoire. Du found footage en général, et des films de Angela Ricci-Lucci et Yervant Gianikian en particulier », Hors Champ, 15 octobre 2006. Accédé : 19 mars 2017. En ligne : http://www.horschamp.qc.ca/spip.php?article226 Voir également : André Habib, «Archives, modes de réemploi. Pour une archéologie du found footage », Cinémas, n²42-243, 2014, pp. 97-122.

18. Marie Rebecchi, « Le temps du remontage : entre cinéma et installation. The Clock de Christian Marcklay », Marges, ${ }^{\circ} 19,2014$, p. 85.

19. Buzz man, « Alain Soral sauve Glenn et Tara dans
The Walking Dead», YouTube, 28 mars 2016. Accédé : 15 février 2017. Disponible : https://www.youtube. com/watch?v=33xsxtSb9zI

20. L'auteur de la vidéo n'a malheureusement jamais donné suite à nos demandes d'entretiens, il nous a fallu nous contenter de données écologiques pour sa mise en contexte et son analyse. Si, comme nous le verrons, il maîtrise l'univers de sens de l'imaginaire complotiste de Soral, nous aurions aimé savoir si l'auteur est militant ou sympathisant d'Égalité \& Réconciliation ou si la vidéo s'inscrit dans une démarche davantage technique, esthétique ou ludique. Nous aurions également souhaiter connaître les dispositifs techniques mobilisés par l'auteur pour réaliser le montage. «Alain Soral sauve Glenn et Tara dans The Walking Dead », jeuxvideo.com, 28 mars 2016. Accédé : mars avril 2017. En ligne $:$ http://www.jeuxvideo.com/forums/4251-46485005-1-0-1-0-alain-soral-sauve-glenn-et-taradans-the-walking-dead.htm

21. Laurent Véray, "Réflexions sur les images d'archives de la Grande Guerre dans les documentaires télévisuels actuels », 1895. Revue de l'association française de recherche sur le cinéma, $\mathrm{n}^{\circ} 64,2011, \mathrm{p} .17$. 22. «Attention les imposteurs : SuperSoral arrive !», Égalité \& Réconciliation, 29 mars 2016. Accédé : 28 mars 2017. En ligne :http://www.egaliteetreconciliation. fr/Attention-les-imposteurs-SuperSoral-arrive-38586. html

23. Série télévisée dramatique ou d'horreur, The Walking Dead est adaptée des bandes dessinées éponymes par Frank Darabont et Robert Kirkman. Sur fond de monde post-apocalyptique, la série met en scène la lutte pour leur survie d'un groupe d'êtres humains face à des zombies menaçants appelés « rôdeurs » ou « marcheurs ».

24. John Cena \& the Trademarc, "The Time is Now», You Can't See Me, Los Angeles, WWE \& Columbia, 2005, 2,58 min. 
25. Pour une vision globale de l'imaginaire complotiste de Soral, voir : Alain Soral, Comprendre l'Empire. Demain la gouvernance globale ou le réveil des nations?, Paris, Éditions Blanche, 2011.

26. Julien Giry, « Le conspirationnisme. Archéologie et morphologie d'un mythe politique », Diogène, $\mathrm{n}^{\circ} 249$ 520, 2015, pp. 40-50.

27. Richard Hofstadter, The Paranoid Style and Other Essays [1964], Cambridge, Harvard University Press, 1996.

28. En référence aux affiches de Jean-Marie Le Pen lors des élections législatives de 1993.

29. Nous soulignons par contraste : armes/mains nues. 30. «Attention les imposteurs : SuperSoral arrive!», op. cit.

31. À l'inverse, les catcheurs heel personnifient la trahison (Un-American), la tricherie, la lâcheté ou l'immoralité. Ils symbolisent les élites corrompues, comme le personnage de texan milliardaire, " roi » du pétrole, JBL lors de la crise de 2008, ainsi que les étrangers subversifs ou couards, le personnage du mollah après le 11 septembre 2001 et l'équipe $L a$ Résistance (en français dans le texte) en 2003 après le refus de la France de participer à la guerre en Irak. 32. Olivier Blondeau, op. cit., pp. 114-116.

33. Nicole Brenez, "Montage intertextuel et formes contemporaines du rempli dans le cinéma expérimental », Cinémas, 2002, vol.13, n ${ }^{\circ} 1-2$, pp. 59-60.

34. David Machin, Adam Jaworski, «Archive Video Footage in News : Creating a Likeness and Index of the Phenomenal World », Visual Communication, vol.5, n³, 2006, p. 346.

35. Olivier Blondeau, op. cit., pp. 119-128.

36. Nicole Brenez, art. cit., pp. 54-57.

37. Laurent Véray, art. cit., p. 27.

38. Olivier Blondeau, op. cit., p. 103.

39. Christine Montalbetti, «Fiction, réel, référence »,
Littérature, $\mathrm{n}^{\circ} 123,2001, \mathrm{p} .49$.

40. Pierre Nora, "Histoire et roman, où passent les frontières ? », Le Débat, vol.3, n¹65, 2011, p. 7.

41. David Machin, Adam Jaworski, art. cit., p. 347.

42. On pense ici aux attaques du 11 septembre ou aux attentats de Charlie Hebdo où, sur la base de seules images, se sont forgées des théories du complot. Sur la question des images et de la vérité, voir : Pierre Lagrange, «Quels arguments opposer aux amateurs de conspirations ? », Mouvements, 2002, vol.5, n²4, pp. 113-119.

43. Howard Besser, "The Internet as De Facto Archive : Communities of Interest Saving Films », Actes du colloque L'Avenir de la mémoire. Patrimoine, restauration et réemploi cinématographiques, Cinémathèque québécoise, Montréal, 13 février 2010. 


\title{
$R \cdot E ́ \cdot S \cdot U \cdot M \cdot E$
}

Cette contribution se propose de procéder à une étude de cas d'une vidéo recombinante (found footage), Alain Soral sauve Glenn et Tara dans The Walking Dead, postée sur YouTube en mars 2016. Nous montrerons qu'il s'agit, à partir d'images de la série télévisée The Walking Dead, d'autres tirées d'un film de propagande pro-Soral et le thème musical du catcheur John Cena, de recréer une nouvelle unité de sens subjective et militante représentative de l'imaginaire conspirationnisme d'Alain Soral. Surtout, par ses usages, cette vidéo nous semble typique du phénomène des vidéos recombinantes qui s'inscrit dans le tournant vidéo du médiactivisme du début des années 2000.

\begin{abstract}
This paper aims to realize a case study of a found footage video, Alain Soral sauve Glenn et Tara dans The Walking Dead, posted on YouTube in march 2016. I argue this video, through footage sacked from the TV show The Walking Dead and a Soral's propaganda movie supported by John Cena's music, creates a new subjective and militant unity of sense that represents symbolically and aesthetically Soral's conspiracist imaginary. By its uses, this video looks typical of the found footage phenomenon which participates of the early 2000's video watershed of mediactivism.
\end{abstract}

